\title{
Preliminary study on the role and mechanism of KIRREL3 in the development of esophageal squamous cell carcinoma (ESCC)
}

Bingbing Yang

Zhengzhou University

Xiane Zhang

Henan Provincial People's Hospital

Hao Zhou

Zhengzhou University

Xiaoyan Zhang

Zhengzhou University

Wanjing Yang

Zhengzhou University

Jing Lu

Zhengzhou University

Zhiyu Guo

SanQuan College of XinXiang Medical University

Ziming Dong

Zhengzhou University

Qinghua Wu

First Affiliated Hospital of Zhengzhou University

Fang Tian ( $\sim$ tianfang418@163.com )

Zhengzhou University

\section{Research Article}

Keywords: Esophageal squamous cell carcinoma, KIRREL3, STAT3, proliferation, invasion

Posted Date: January 13th, 2022

DOI: https://doi.org/10.21203/rs.3.rs-844973/v2

License: (1) (1) This work is licensed under a Creative Commons Attribution 4.0 International License.

Read Full License 


\title{
Preliminary study on the role and mechanism of KIRREL3 in the development of esophageal squamous cell carcinoma (ESCC)
}

\author{
Bingbing Yang ${ }^{1,2,3 \#}$, Xiane Zhang ${ }^{1,2,4 \#}$, Hao Zhou ${ }^{1,2,3}$, Xiaoyan Zhang \\ 1,2,3, Wanjing Yang ${ }^{1,2,3}$, Jing $\mathrm{Lu}^{1,2,3}$, Zhiyu Guo ${ }^{5}$, Ziming Dong ${ }^{1,2,3}$, \\ Qinghua $\mathrm{Wu}^{6}$ and Fang Tian ${ }^{1,2,3 *}$
}

\# Co-first author

* Correspondence: tianfang418@ 163.com

${ }^{1}$ The Pathophysiology Department, School of Basic Medical Sciences,

Zhengzhou University, Zhengzhou 450001, China; ${ }^{2}$ Provincial Cooperative

Innovation Center for Cancer Chemoprevention, Zhengzhou University,

Zhengzhou 450001, China; ${ }^{3}$ Cancer Chemoprevention International

Collaboration Laboratory, Zhengzhou 450001, China; ${ }^{4}$ Second People's

Hospital of Henan Province, Zhengzhou 451191, China;

Full list of author information is available at the end of the article

\begin{abstract}
Background: Esophageal squamous cell carcinoma (ESCC) is a common malignant tumor of the digestive tract, which is very harmful to human health. The JAK-STAT signaling pathway is a recognized carcinogenic pathway that plays a role in the proliferation, apoptosis, migration, and invasion of a variety of cancer cells. Some studies have shown that the activation status of STAT3 affects the expression of KIRREL3. However, the expression of KIRREL3 in ESCC and its relationship with KIRREL3 or the JAK-STAT signaling pathway is still unclear.
\end{abstract}

Methods: In this study, we used immunohistochemistry and western blotting to analyze the protein expression levels of KIRREL3 in tumor tissues and ESCC cell lines. We applied 
proliferation assays, plate clone formation assays, Transwell assays, flow cytometry analysis, and CDX animal models to examine the role of KIRREL3 in ESCC.

Results: The results indicate that KIRREL3 is highly expressed to varying degrees in ESCC tissues and cell lines. Knocking down KIRREL3 expression in ESCC cells could correspondingly inhibit cell proliferation, colony formation, invasion, and migration, and had some effects on cell cycle progression and apoptosis. In addition, overexpressing KIRREL3 in these cells had opposite effects. Tumor formation in nude mice experiments also confirmed that KIRREL3 is involved in the growth of ESCC cells in vivo.

Conclusions: These data suggest that KIRREL3 plays a key role in the development of ESCC, and KIRREL3 is a potential new target for the early diagnosis and clinical treatment of this disease.

Keywords: Esophageal squamous cell carcinoma, KIRREL3, STAT3, proliferation, invasion

\section{Background}

Esophageal cancer is the seventh most common cancer and the sixth most common cause of cancer-related death in the world ${ }^{[1]}$. Its major histological types are esophageal adenocarcinoma (EAC) and esophageal squamous cell carcinoma (ESCC) ${ }^{[2]}$. EAC is the main histological type in western countries $^{[3][4]}$, while ESCC is a common histological type in Asian and African countries ${ }^{[5]}$ and accounts for about $90 \%$ of all esophageal cancer cases in China ${ }^{[6][7]}$. Poor prognosis and higher recurrence rates result in higher mortality in ESCC patients ${ }^{[8][9]}$. Although there are various treatment methods for ESCC, such as surgical chemotherapy and radiotherapy, they are not fully effective $^{[10]}$. Therefore, there is an urgent need to explore new therapeutic targets to inhibit tumor progression in ESCC patients.

The Janus kinase-signal transducers and activators of transcription (JAK-STAT) pathway plays an important biological role in promoting tumor cell survival, proliferation, inflammation, invasion, and metastasis ${ }^{[11][12]}$. STAT3, the most important member of the STAT family, is frequently activated in inflammation and cancer ${ }^{[13][14]}$. After activation, it forms a homodimer and is transported to the nucleus to bind DNA, thus promoting the transcription of genes related to anti-apoptosis, invasion, and migration ${ }^{[13][15]}$. STAT3 is currently known to be co-expressed with multiple oncogenes ${ }^{[16][17]}$. For example, researchers have found that the expression levels of KIRREL3 (Kin of Irre-like 3) are correlated with the activation level of STAT3 ${ }^{[18][19] .}$ 
Previous work from this group showed that KIRREL3 is an immunoglobulin superfamily adhesion molecule and is a transmembrane protein ${ }^{[20]}$. KIRREL3 protein contains five immunoglobulin-like domains, a single transmembrane domain, and a cytoplasmic domain in the extracellular region ${ }^{[21]}$. Studies have shown that point mutations and deletions of the KIRREL3 gene are associated with neurodevelopmental disorders, such as autism, mental retardation, and Jacobson syndrome ${ }^{[22]}$. Currently, there is little literature describing KIRREL3 in tumors. Therefore, we aimed to investigate the role of KIRREL3 in ESCC.

In this study, we found that KIRREL3 had different degrees of high expression in ESCC tissues and cells. Downregulating KIRREL3 expression could inhibit cell proliferation, migration, and invasion, while overexpression of KIRREL3 could promote cell proliferation, migration, and invasion. KIRREL3 knockdown had some effects on cell cycle and apoptosis. In addition, tumor formation experiments in nude mice demonstrated that KIRREL3 is involved in ESCC tumor growth. These data suggest that KIRREL3 plays an important role in the proliferation and metastasis of ESCC, making it a possible new target for the early diagnosis and clinical treatment of this disease.

\section{Methods}

\section{Reagents}

A small interfering RNA (siRNA) targeting KIRREL3 and scrambled control were purchased from GenePharma (Shanghai, China). Lipofectamine ${ }^{\circledR} 2000$ was purchased from Invitrogen (Carlsbad, CA, USA). The overexpression lentivirus and shRNA lentivirus were purchased from GenePharma. The primary antibodies against KIRREL3 were purchased from Abcam (Cambridge, UK). The primary antibodies against phospho-STAT3 (Tyr705) and STAT3 were purchased from Cell Signaling Technology (Danvers, MA, USA). Anti- $\beta$-Actin, anti-mouse $\mathrm{IgG}$, and anti-rabbit $\mathrm{IgG}$ horseradish peroxidase-conjugated antibodies were purchased from ZhongshanJinqiao (Beijing, China). Curcumin was purchased from Selleck Chemicals (Houston, TX, USA). STAT3 inhibitor Stattic was purchased from Sigma (St. Louis, MO, USA).

\section{Immunohistochemistry (IHC)}

Tissues were dehydrated with xylene and an ethanol series, followed by washes with TBST. Antigen retrieval was performed with sodium citrate $(0.01 \mathrm{M}, \mathrm{pH}=6.0)$. Endogenous 
peroxidase was blocked by using $\mathrm{H}_{2} \mathrm{O}_{2}$ for 10 minutes at room temperature. After washing with TBST, the KIRREL3 primary antibody (LifeSpan BioSciences, Seattle, WA, USA, 1:100) was added and tissues were incubated overnight at $4^{\circ} \mathrm{C}$. They were then incubated with secondary antibody at $37^{\circ} \mathrm{C}$ for 15 minutes. They were visualized by incubating with $\mathrm{DAB}$, and staining was observed with microscopy. After decoloring with $1 \%$ hydrochloric acid ethanol, the tissues were dehydrated and transparentized by ethanol and xylene. The tissues were sealed by neutral resin.

\section{Cell lines and cell culture}

Immortalized human esophageal epithelial SHEE and ESCC cell lines (Eca109, KYSE150, KYSE30, KYSE450, and KYSE510) were provided by the Department of Pathophysiology, School of Basic Medicine, Zhengzhou University. Eca109 and KYSE150 cells were cultured in RPMI 1640 medium supplemented with 10\% fetal bovine serum (FBS) (BI, Kibbutz Beit Haemek, Israel). KYSE30, KYSE450, and KYSE510 cells were cultured in DMEM supplemented with $10 \%$ FBS. All of these cells were maintained at $37^{\circ} \mathrm{C}$ in a $5 \% \mathrm{CO}_{2}$ environment.

\section{siRNA transfections}

Lipofectamine ${ }^{\circledR} 2000$ reagent was used for all siRNA transfection procedures at a final concentration of $10 \mu \mathrm{M}$. For immunoblotting analysis, $6.5 \times 10^{5}$ Eca109 cells and $5 \times 10^{5}$ KYSE150 cells were seeded into 6-well plates and incubated overnight. They were transfected with siRNAs for 72 hours and then collected. For apoptosis assays, Eca109 and KYSE150 cells were seeded into 6-well plates and incubated overnight. After siRNA transfections, the cells were cultured for 48 hours and collected for apoptosis analysis.

\section{Lentivirus infections}

To establish stable cell lines that overexpressed or knocked down KIRREL3, we obtained appropriate lentiviruses from GenePharma. Transfections of cells were done according to manufacturer instructions. After target cells were infected, stably transfected cells were obtained by screening with puromycin $(1 \mu \mathrm{g} / \mathrm{mL}$ for Eca109 cells; $2 \mu \mathrm{g} / \mathrm{mL}$ for KYSE150 and KYSE30 cells).

\section{Immunoblotting analysis}


Cells were washed two or three times with ice-cold phosphate-buffered saline (PBS). Proteins were extracted with protein lysis buffer. Protein concentrations were measured with the BCA Protein Concentration Kit (Solarbio, Beijing, China). Samples were separated by $10 \%$ polyacrylamide gel electrophoresis (SDS-PAGE) and transferred to a PVDF membrane. The membrane was incubated with primary antibody overnight ( $\sim 16$ hours) at $4^{\circ} \mathrm{C}$. The primary antibodies included KIRREL3 (Abcam, 1:1000), p-STAT3 (Tyr705) (Cell Signaling Technology, 1:1000), STAT3 (Cell Signaling Technology, 1:1000), and $\beta$-actin (Zhongshan jinqiao, 1:1000).

\section{Cell proliferation assay}

Cells $(5,000$ cells/well $)$ were seeded into 96-well plates and cultured overnight. Proliferation was measured by CCK-8 assay. After being cultured for $0,24,48$, 72, or 96 hours, $10 \mu \mathrm{L}$ of CCK8 reagent (Beyotime, Shanghai, China) was added to each well. Following incubation for 1 hour, absorbance values at $450 \mathrm{~nm}$ were measured.

\section{Cell colony formation assay}

Eca109 and KYSE150 cells were seeded into 6-well plates at 300 cells/well and KYSE30 cells were seeded into 6-well plates at 400 cells/well. The cells were cultured for one to two weeks until colonies appeared. The medium was then removed and the cells were washed with PBS, followed by fixation in each well with $4 \%$ paraformaldehyde (PFA) for 30 minutes. Finally, cells were stained with $0.1 \%$ crystal violet $(\mathrm{CV})$ for 30 minutes.

\section{Transwell cell invasion and migration assays}

Cell invasion ability was evaluated by Transwell cell invasion assays. An invasion assay was performed using Transwell Matrigel (Corning, Corning, NY, USA). Cells ( $5 \times 10^{5}$ cells/well for KYSE150 and Eca109 and $3 \times 10^{5}$ cells/well for KYSE30) were seeded into the upper chamber of the plates in RPMI 1640 or DMEM (serum-free) after siRNA transfection or lentivirus infection. RPMI 1640 or DMEM with 10\% FBS was then added to the lower chamber. After $30-40$ hours of incubation at $37^{\circ} \mathrm{C}$ with $5 \% \mathrm{CO}_{2}$, the Matrigel and cells in the upper chamber were discarded.

The medium was removed and the cells were washed with PBS, followed by fixation in each well with 4\% PFA for 30 minutes. Finally, cells were stained with $0.1 \%$ CV for 30 minutes. Transwell plates without Matrigel were used for migration assays, with the rest of the procedure remaining the same. 


\section{Cell cycle assay}

Cells were added to $75 \%$ ethyl alcohol that was pre-cooled overnight. After washing the cells once with PBS, $1 \mathrm{ml}$ PBS was added to resuspend the cells. Then, $2.5 \mu \mathrm{L}$ RNase (10 $\mathrm{mg} / \mathrm{mL}$ ) (Meilunbio, Dalian, China) was added and incubated at room temperature for 1 hour. Finally, $50 \mu \mathrm{L}$ propidium iodide (PI) (1 mg/mL) (Meilunbio) was added and incubated at room temperature or $4^{\circ} \mathrm{C}$ in the dark for $15-20$ minutes. The cells were analyzed using flow cytometry.

\section{Cell apoptosis assay}

Apoptosis levels were detected 48 hours after siRNA transfections. The cells were stained with an Annexin V-FITC/PI kit (Meilunbio). Cells were digested with trypsin without EDTA and washed with PBS. The cells were resuspended with $100 \mu \mathrm{L} 1 \times$ Binding buffer, then $5 \mu \mathrm{L}$ Annexin V-FITC and $6 \mu \mathrm{L}$ PI were added to the cell suspension, mixing well and protected from light for 20 minutes at room temperature. The proportion of early or late apoptotic cells was measured by flow cytometry.

\section{Tumor xenograft experiments}

Female BALB/c nude mice were purchased from the Beijing Weitonglihua (Beijing, China). Four-week-old female mice were maintained in specific pathogen-free conditions. The nude mice were subcutaneously injected with $5 \times 10^{7}$ Eca109 or KYSE-150 cells stably transfected with KIRREL3 or the empty vector. The mice weight and tumor growth level were measured three times a week. Tumor volume was calculated as length $\times w_{i d t h}{ }^{2} \times 1 / 2$.

\section{Statistical analysis}

All statistical analyses were performed using SPSS version 17.0 (SPSS, Chicago, IL, USA). The data were analyzed by one-way analysis of variance (ANOVA) or Student $t$ test. Data are expressed as means \pm standard deviation (SD). The statistically significant criterion was $\mathrm{p}<0.05$.

\section{Results}

\section{KIRREL3 protein expression levels are increased in ESCC tissues and cell lines}

The protein expression levels of KIRREL3 in ESCC tissues were detected by IHC. We observed high expression of KIRREL3 in 56.76\% (42/74) of ESCC tissues. Compared with $31.88 \%$ (22/69) of adjacent normal esophageal tissues, KIRREL3 was significantly 
overexpressed in ESCC tissues (Table 1; Figure 1A). We analyzed the relationship between KIRREL3 expression and clinical parameters and found that the overexpression of KIRREL3 was negatively correlated with the degree of tissue differentiation (Table 2).

We further analyzed the expression of KIRREL3 in normal esophageal epithelial cells and ESCC cell lines with immunoblots. KIRREL3 protein expression levels in KYSE150, KYSE30, KYSE450, Eca109, and KYSE510 cells were higher than that of SHEE cells (Figure 1B).

Table 1: Expression scores were quantified in normal adjacent tissues and ESCC tumor samples.

\begin{tabular}{|c|c|c|c|c|c|}
\hline \multirow{2}{*}{ histologic type } & \multirow{2}{*}{$\mathrm{n}$} & \multicolumn{2}{|c|}{$\begin{array}{l}\text { Expression of KIRREL3 in } \\
\text { tissue }\end{array}$} & \multirow{2}{*}{$X^{2}$} & \multirow{2}{*}{ palue } \\
\hline & & + & - & & \\
\hline ESCC & 74 & 42 & 32 & 8.934 & 0.003 \\
\hline adjacent tissues & 69 & 22 & 47 & & \\
\hline
\end{tabular}

$\mathrm{A}_{20 \mathrm{x}_{\mathrm{a}}}$
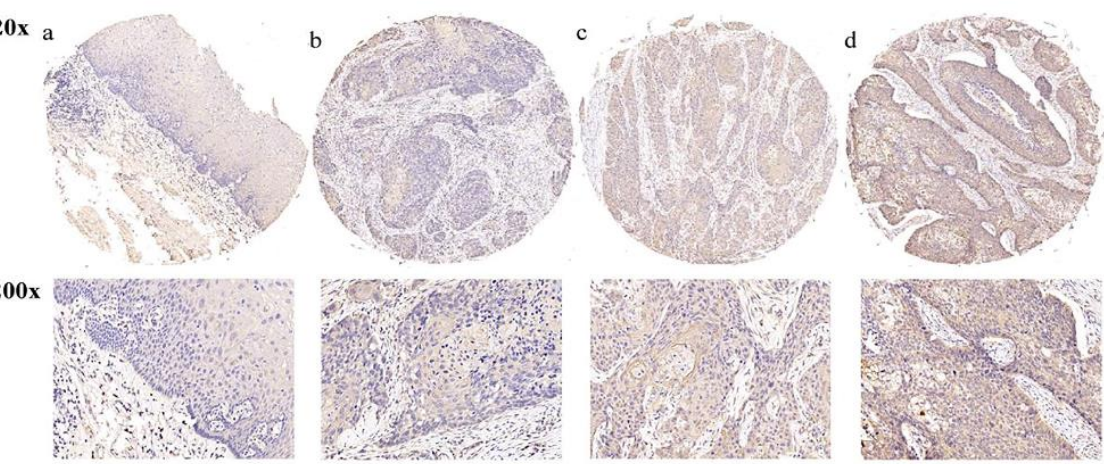

B

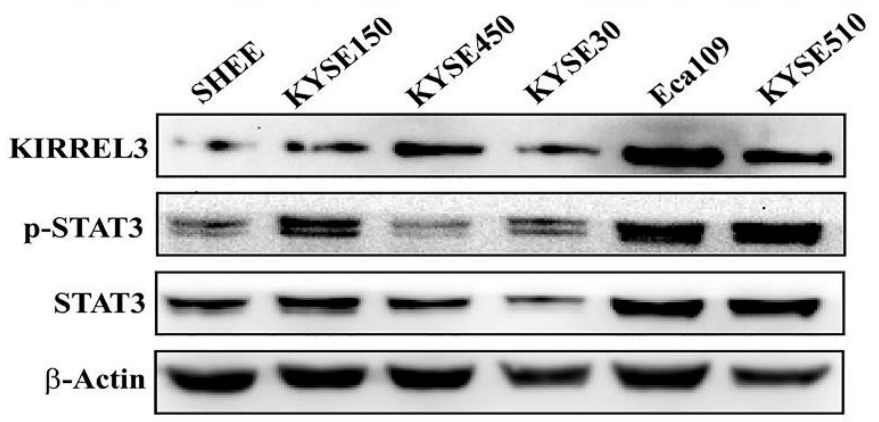

Figure 1. KIRREL3 protein expression in esophageal squamous cell carcinoma (ESCC) tissues and cell lines. (A) Representative KIRREL3 immunohistochemistry (IHC) images in highly differentiated, moderately differentiated, and poorly differentiated tumor and normal adjacent 
tissues. Expression scores were quantified in adjacent tissues $(n=69)$ and tumor $(n=74)$ samples (Table 1). (B) Protein expression of KIRREL3 in ESCC cells and esophageal epithelial immortalized cells by immunoblot analysis.

Table2: The association between KIRREL3 levels and clinicopathological features in tumor samples.

\begin{tabular}{|c|c|c|c|}
\hline \multirow[t]{2}{*}{ Variables } & \multicolumn{3}{|c|}{ KIRREL 3 expression ( $\mathrm{n}=74$ cases) } \\
\hline & Low $(\%)$ & High $(\%)$ & $P^{a}$ \\
\hline All patients & $32(100)$ & $42(100)$ & \\
\hline Age(years) & & & 0.971 \\
\hline$\leqq 65$ & $23(74)$ & $31(74)$ & \\
\hline$>65$ & $8(26)$ & $11(26)$ & \\
\hline Gender & & & 0.276 \\
\hline Males & $20(65)$ & $32(76)$ & \\
\hline Females & $11(35)$ & $10(24)$ & \\
\hline Depth of invasion & & & 0.416 \\
\hline $\mathrm{T} 1 / \mathrm{T} 2$ & $12(50)$ & $15(39)$ & \\
\hline $\mathrm{T} 3 / \mathrm{T} 4$ & $12(50)$ & $23(61)$ & \\
\hline Lymph node metastasis & & & 0.770 \\
\hline No & $11(37)$ & $14(33)$ & \\
\hline $\mathrm{N} 1 / \mathrm{N} 2 / \mathrm{N} 3$ & $19(63)$ & $28(67)$ & \\
\hline Distant metastasis & & & 0.211 \\
\hline M0 & $28(88)$ & $41(98)$ & \\
\hline M1 & $4(12)$ & $1(2)$ & \\
\hline \multicolumn{4}{|l|}{ TNM stage } \\
\hline I & $3(9)$ & $4(10)$ & 0.313 \\
\hline II & $9(28)$ & $17(40)$ & \\
\hline III & $16(50)$ & $20(48)$ & \\
\hline IV & $4(13)$ & $1(2)$ & \\
\hline Differentiation & & & $0.024 *$ \\
\hline Poor & $7(22)$ & $19(45)$ & \\
\hline Moderate & $22(69)$ & $23(55)$ & \\
\hline High & $3(9)$ & & \\
\hline
\end{tabular}




\section{KIRREL3-regulated cell proliferation and the cell cycle in ESCC cells}

We next examined the effect of KIRREL3 on ESCC cell proliferation. The shRNA vector, siRNAs, and overexpression vector were transfected into ESCC cells (Figure 2A). CCK8 assays suggested that KIRREL3 overexpression significantly increased ESCC cell proliferation, but KIRREL3 knockdown significantly decreased proliferation (Figure 2B, 2C, 2D). Colony formation assays showed that overexpression of KIRREL3 enhanced the colony formation ability of ESCC cells (Figure 3C). In contrast, KIRREL3 knockdown inhibited the proliferation of ESCC cells, as seen by reduced colony formation (Figure 3A, 3B). For cell cycle analysis, depleting KIRREL3 levels increased the number of Eca109 and KYSE150 cells in G2/M arrest (Figure 3D). These results indicate that KIRREL3 is involved in regulating the proliferation and cell cycle progression of ESCC cells.

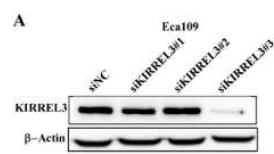

B

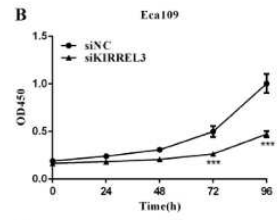

D

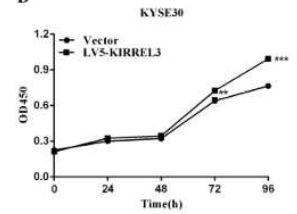

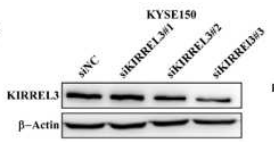
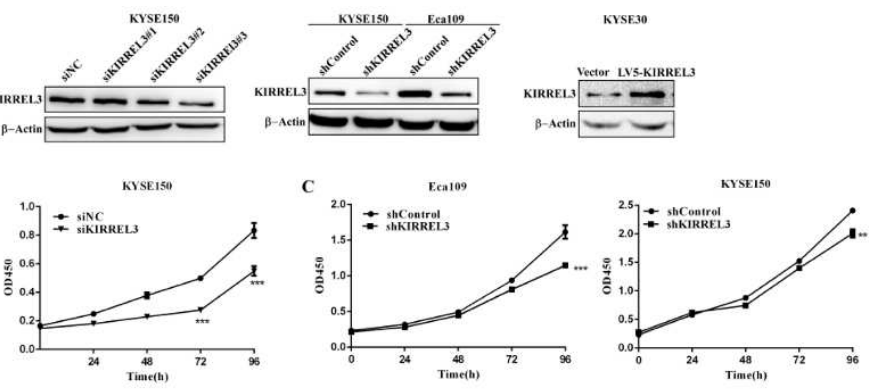

Figure 2. KIRREL3-mediated regulation of esophageal squamous cell carcinoma (ESCC) cell proliferation measured by CCK8 assays. (A) Measurement of the efficacy of KIRREL3 knockdown and overexpression via western blotting in Eca109, KYSE150, and KYSE30 cells. Evaluation of the proliferation abilities of (B) Eca109, (C) KYSE150, and (D) KYSE30 cells via CCK8 proliferation assays. ${ }^{* *} p<0.01, * * * p<0.001$. 


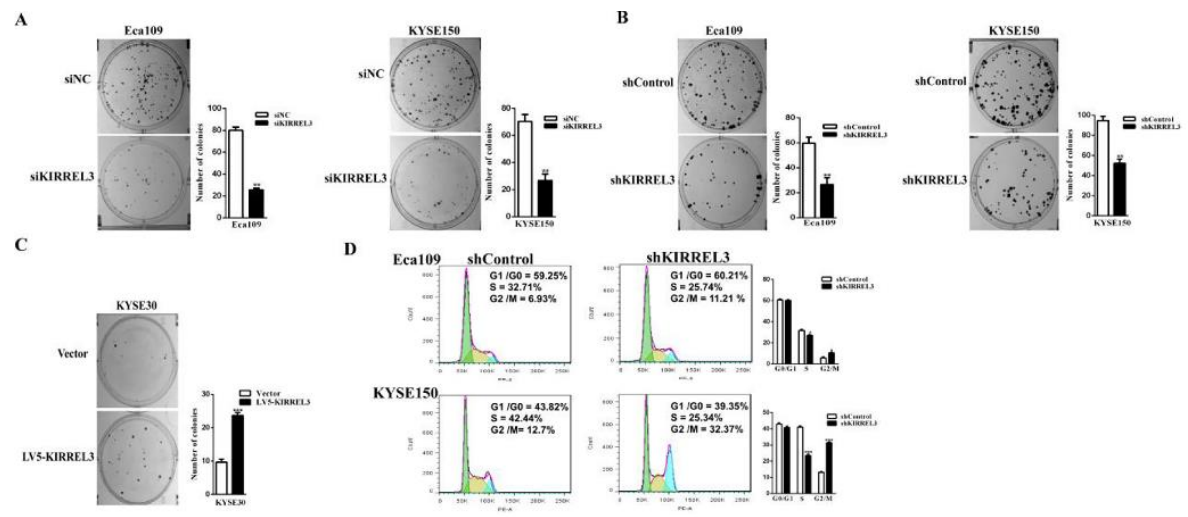

Figure 3. KIRREL3-mediated regulation of esophageal squamous cell carcinoma (ESCC) cell proliferation measured by colony formation assays and cell cycle analysis. Evaluation of the proliferation abilities of (A) Eca109, (B) KYSE150, and (C) KYSE30 cells via colony formation assays. (D) Measurement of cell cycle progression in Eca109 and KYSE150 cells transfected with siKIRREL3 or siNC via flow cytometry. ${ }^{*} p<0.05, * * p<0.01,{ }^{* * *} p<0.001$.

\section{KIRREL3-mediated regulation of apoptosis in ESCC cells}

To investigate the role of KIRREL3 in cell survival, the KIRREL3-targeting siRNA was transfected into KYSE150 and Eca109 cells, followed by apoptosis analysis. The depletion of KIRREL3 significantly increased apoptosis levels in both the KYSE150 and Eca109 cells 48 hours after siRNA transfection (Figure 4A, 4B).

A
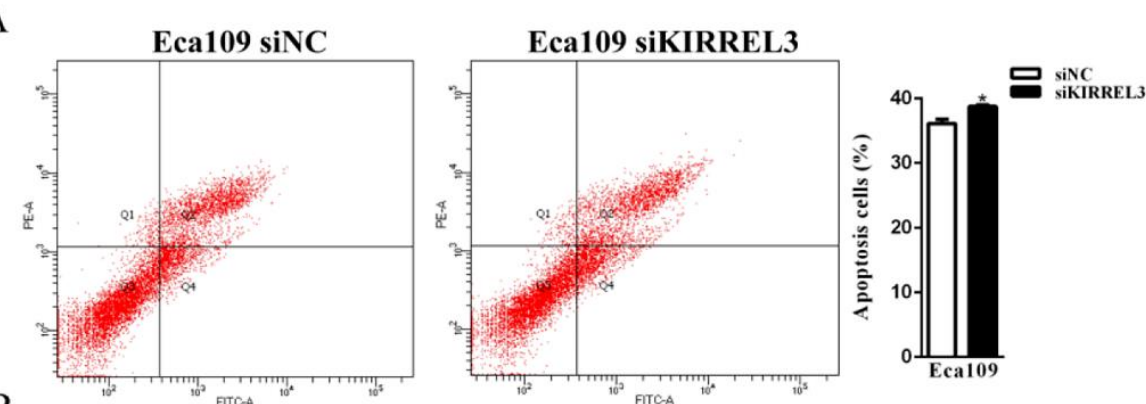

B
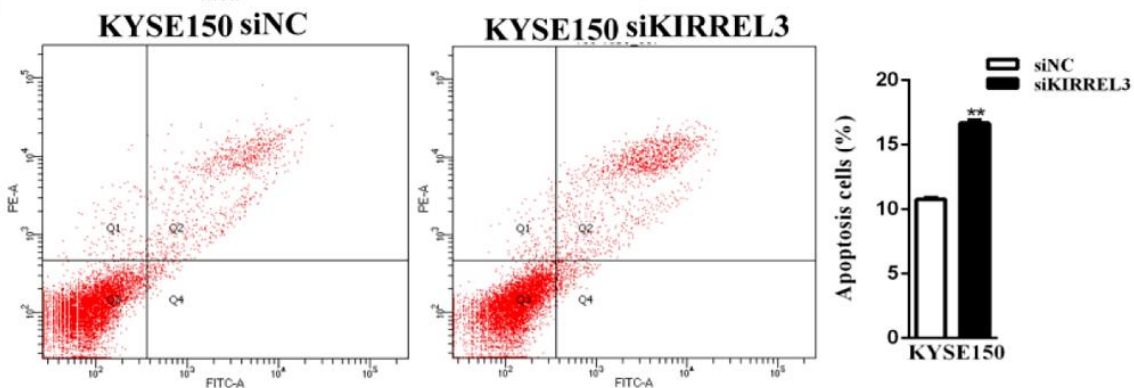

Figure 4. KIRREL3 knockdown induces apoptosis in Eca109 and KYSE150 cells. Following siRNA transfection, the apoptosis levels of (A) Eca109 and (B) KYSE150 cells were evaluated by flow cytometry. $* p<0.05, * * p<0.01$. 


\section{KIRREL3 promotes invasion and migration of ESCC cells}

The effects of KIRREL3 on invasion and migration rates were investigated in ESCC cells. The Transwell experiment results suggested that KIRREL3 could promote cell invasion and migration, as the numbers of invading and migrating cells increased following overexpression of KIRREL3 (Figure 5C), while the number of invading and migrating cells decreased after KIRREL3 knockdown (Figure 5A, 5B).
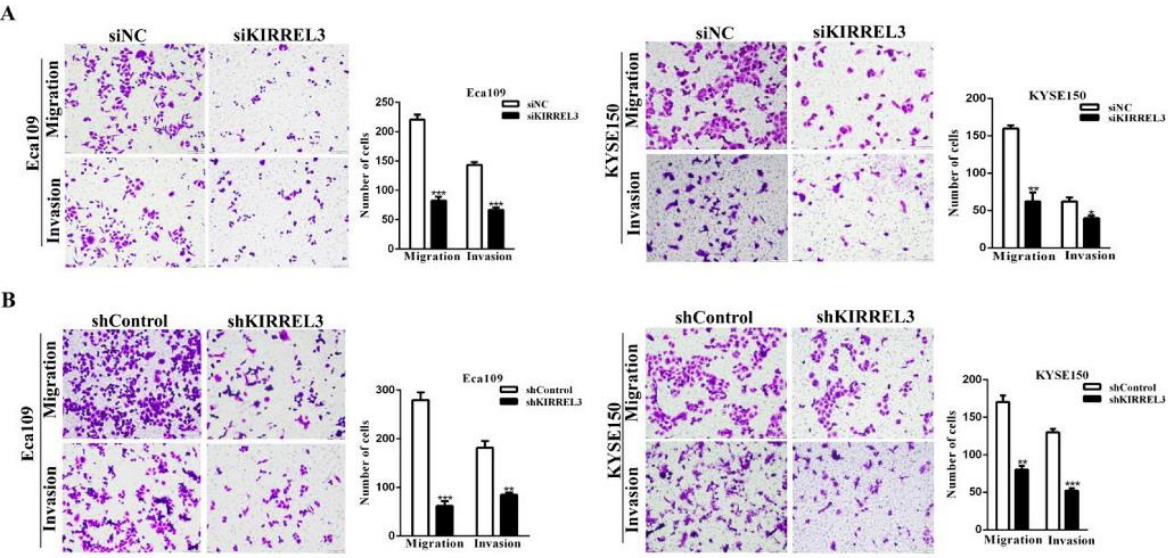

C

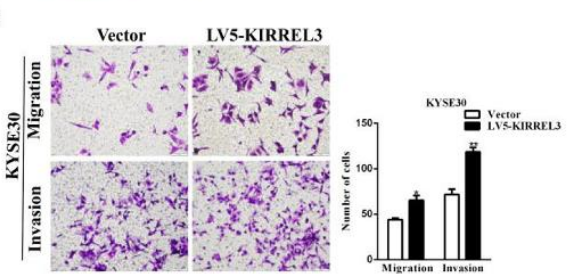

Figure 5. KIRREL3 promotes the invasion and migration of esophageal squamous cell carcinoma (ESCC) cells. The (A) invasion and (B) migration of siRNA-transfected Eca109 and KYSE150 cells were evaluated by Transwell assays. (C) The invasion and migration of KYSE30 cells overexpressing KIRREL3 were evaluated by Transwell assays. ${ }^{*} p<0.05$, ${ }^{* *} p$ $<0.01, * * * p<0.001$.

\section{STAT3 may regulate KIRREL3}

We explored the relationship between STAT3 and KIRREL3 in Eca109 and KYSE150 cells that express hyperphosphorylated STAT3. The small molecule STAT3 inhibitor Stattic has cell permeability and can block STAT3 activation. Therefore, we examined its effects on Eca109 and KYSE150 cells. Stattic dose-dependently inhibited the levels of p-STAT3 in Eca109 and KYSE150 cells. KIRREL3 protein expression levels did not change significantly, but was suppressed at the maximum concentration of Stattic (Figure 6B). Curcumin is an 
indirect natural inhibitor of STAT3. In Eca109 and KYSE150 cells, curcumin dosedependently inhibited p-STAT3 and KIRREL3 protein levels (Figure 6A). These results suggest that the expression levels of KIRREL3 correlated with the phosphorylation of pSTAT3.
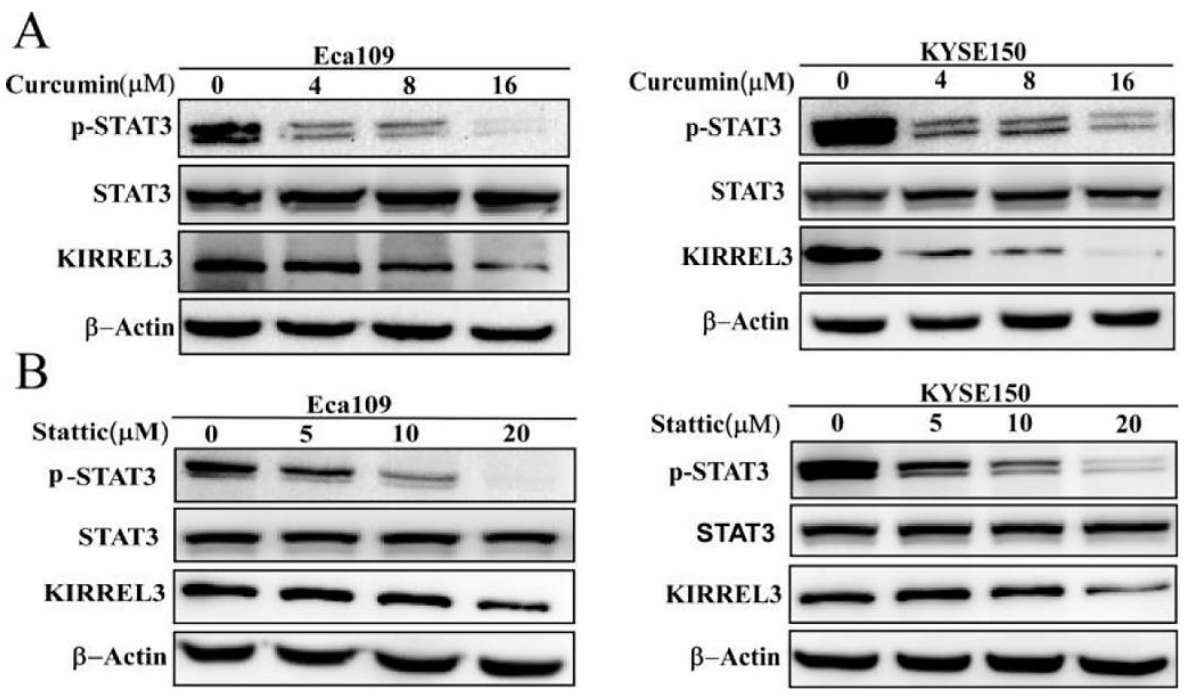

Figure 6. The relationship between KIRREL3 and STAT3 was examined by western blotting. (A) The protein expression levels of p-STAT3 and KIRREL3 were dose-dependently inhibited by curcumin treatment of Eca109 and KYSE150 cells. (B) The protein expression levels of pSTAT3 were dose-dependently inhibited by Stattic treatment of Eca109 and KYSE150 cells. Protein expression of KIRRREL3 was also inhibited under the maximum concentration of Stattic.

\section{Silencing KIRREL3 expression inhibits tumor growth in vivo}

To assess the effect of KIRREL3 expression on ESCC cell growth in vivo, we established a Cancer cell line-based xenograft (CDX) animal model of KIRREL3 with KYSE150shControl, KYSE150-shKIRREL3, Eca109, Eca109-shControl, or Eca109-shKIRREL3 cell lines in BABL/c nude mice. After KYSE150-shControl and KYSE150-shKIRREL3 cells formed tumors, the tumor growth was monitored periodically and tumor tissues were collected at the end of the experiment. The tumor growth curve data shows that the tumor growth of the KYSE150-shKIRREL3 group was significantly slower than that of the control group (Figure 7A). Additionally, the average weight of KYSE150-shKIRREL3 tumors was less than that of the control group. The difference in tumor weight was significant between the two groups (Figure 7A). 
After Eca109-shControl and Eca109-shKIRREL3 cells formed tumors, the tumor growth was monitored periodically. After Eca109 cells formed tumors, they were randomly divided into three groups. Mice were treated with siNC, siKIRREL3, and Stattic, and the Stattic prevention group was given a week in advance. Tumor tissues were collected after the experiment. The tumor growth curve data suggest that the tumor growth of the siKIRREL3 group was lower than that of the siNC group (Figure 7B). Furthermore, the average weight of tumors in the siKIRREL3 group was lower than that of the siNC group (Figure 7B). For the Eca109-shControl, Eca109-shKIRREL3, and Stattic prevention groups, the tumor growth curve results suggest that the tumor growth of the Eca109-shKIRREL3 and Stattic prevention groups were lower than that of the Eca109-shControl group (Figure 7C). The average weight of tumors in the Eca109-shKIRREL3 and Stattic prevention groups were lower than that of the Eca109shControl group (Figure 7C).

We further analyzed the tumor tissues in the Eca109-shControl, Eca109-shKIRREL3, siNC, siKIRREL3, and Static prevention groups. Compared with the respective control group, the KIRREL3 knockdown group had lower KIRREL3 expression, while p-STAT3 phosphorylation was not affected. Compared with the control group, KIRREL3 and p-STAT3 phosphorylation in the Stattic prevention group were significantly reduced (Figure 7D). In summary, KIRREL3 is involved in the growth of tumor cells in vivo, and KIRREL3 is likely located downstream of STAT3. 

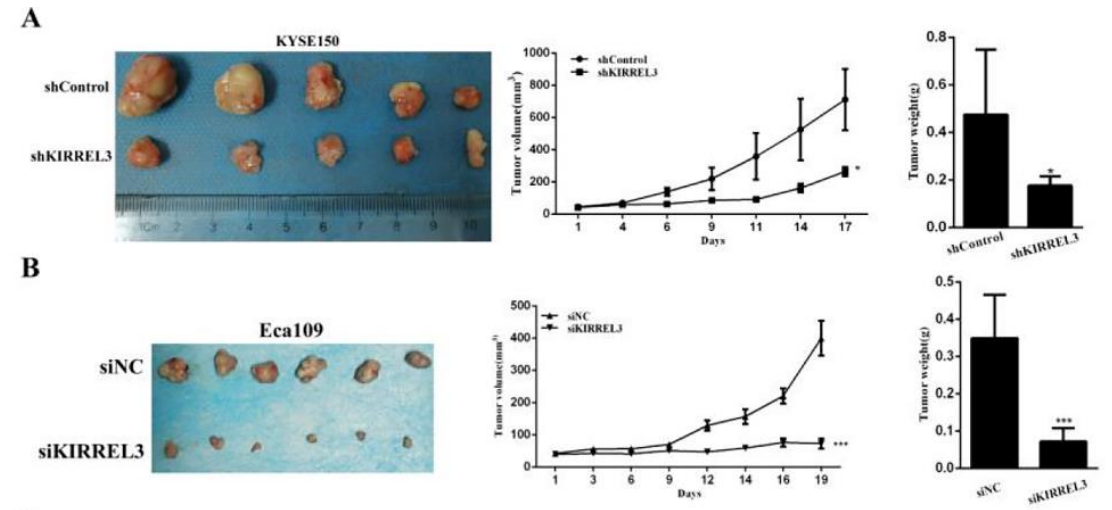

C
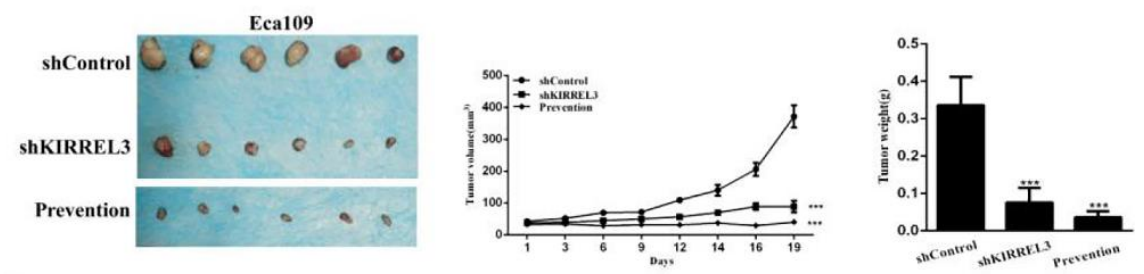

D

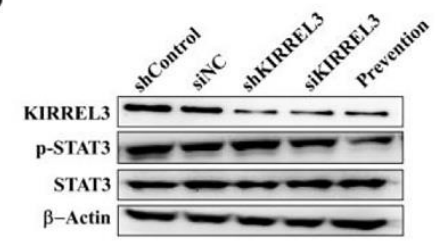

Figure 7. Silencing KIRREL3 inhibits esophageal squamous cell carcinoma (ESCC) tumor growth in vivo. Photographs of tumors from each group were taken, their weights were measured, and tumor volumes in each group was monitored during the experiment. (A) CDX mouse model results with KYSE150-shControl and KYSE150-shKIRREL3 cells. (B) CDX mouse model results with Eca109 cells. Six mice bearing the CDX were treated with siNC or siKIRREL3. (C) The CDX mouse model was constructed with Eca109-shControl and Eca109shKIRREL3 cells. Six mice bearing the CDX were treated with Stattic. $* p<0.05, * * * p<0.001$.

\section{Discussion}

Esophageal cancer is a malignancy with high mortality and low prognosis rates worldwide ${ }^{[24]}$. More than $50 \%$ of the newly diagnosed cases occurred in China ${ }^{[25]}$, with the main pathological type being ESCC ${ }^{[26]}$. The poor prognosis of ESCC is mainly because of the absence of obvious symptoms in the early stage of ESCC, resulting in the disease being frequently diagnosed as advanced ${ }^{[27][28]}$. Therefore, it is urgent to determine the mechanism controlling ESCC development and to identify new clinical diagnostic and therapeutic targets.

The classic JAK-STAT pathway regulates many genes that play key roles in several cancer hallmarks, including uncontrolled cell proliferation, evasion of apoptosis, invasion, angiogenesis, and immune escape ${ }^{[29][32]}$. Studies have shown that the expression of KIRREL3 
in ESCC may be regulated by STAT3. At present, there is no relevant literature on KIRREL3 in ESCC or its relationship with STAT3. Therefore, we focused on the role of KIRREL3 in the development of ESCC, as well as any correlation between KIRREL3 and STAT3.

We examined KIRREL3 expression levels in ESCC tissues and analyzed clinical data. The results showed that KIRREL3 protein was highly expressed in ESCC tissues, and its expression levels were negatively correlated with the degree of tumor differentiation. KIRREL3 is also highly expressed in ESCC cell lines to varying degrees. Based on these data, we chose KYSE150 and Eca109 cells for further experiments. KIRREL3 expression was knocked down using siRNA interference technology and lentiviral infection experiments, resulting in inhibited levels of proliferation in ESCC cells. The cell cycle and cellular evasion of apoptosis are closely related to cell proliferation. An imbalance in their control mechanisms can change cell growth and promote uncontrolled abnormal proliferation of tumor cells ${ }^{[32][33]}$. Our results suggest that KIRREL3 knockdown blocked cell cycle progression and promoted apoptosis. The difficulties associated with treating recurrent malignant tumors and the resulting low survival rates of patients are closely related to the easy invasion and metastasis of these tumor cells ${ }^{[34][35]}$. Transwell assays were used to determine whether KIRREL3 expression was related to ESCC cell invasion and migration. The results showed that the invasion and migration abilities of ESCC cells were reduced following KIRREL3 knockdown.

To further verify the role of KIRREL3 in ESCC cells, we overexpressed KIRREL3 in KYSE30 cells by lentiviral infection. The functional changes of ESCC cells were detected by the same experiments, and the results showed that the proliferation, invasion, and migration of ESCC cells were enhanced with KIRREL3 upregulation.

Studies have reported that abnormal activation of the JAK-STAT signaling pathway can promote tumorigenesis ${ }^{[36]}$. The expression of KIRREL3 may be regulated by STAT3. In this study, we further explored the relationship between KIRREL3 and STAT3. Different concentrations of Stattic and curcumin were added to KYSE150 and Eca109 cells. The results showed that the expression levels of p-STAT3 and KIRREL3 were significantly lower with increasing curcumin concentration. Following Stattic treatment of cells, the expression of $p$ STAT3 was gradually inhibited with increased concentrations. While the downregulation of KIRREL3 was not very robust, it was inhibited at the maximum concentration of Stattic that we used. These data suggest that both curcumin and Stattic can affect the expression of KIRREL3 by downregulating protein levels of p-STAT3. KIRREL3 may be located downstream in the JAK-STAT pathway, which is often abnormally activated and plays a regulatory role in the development of ESCC. 
By constructing a CDX mouse model, we also found that KIRREL3 is involved in ESCC cell proliferation in vivo. The results showed that both downregulation of KIRREL3 and inhibition of p-STAT3 could inhibit the growth of ESCC tumor tissues in mice.

\title{
Conclusion
}

Our study shows that KIRREL3 is highly expressed in ESCC tissues and cell lines to varying degrees. Both in vivo and in vitro experiments have confirmed that KIRREL3 plays an important role in the proliferation, invasion, migration, and apoptosis evasion of ESCC, and KIRREL3 may participate in the JAK-STAT signaling pathway to promote the occurrence and development of esophageal cancer.

\begin{abstract}
Abbreviations
ESCC: Esophageal squamous cell carcinoma; EAC: adenocarcinoma; JAK-STAT: The Janus kinase-signal transducers and activators of transcription; KIRREL3: Kin of Irrelike 3; SiRNA: small interfering RNA; IHC: Immunohistochemistry; CDX: Cancer cell line-based xenograft.
\end{abstract}

\section{Acknowledgements}

Not applicable.

\section{Authors' Contributions}

ZXE, YBB, ZH, ZXY, YWJ, GZY, LJ, WQH and DZM performed the experiments. ZXE, YBB, and TF wrote and edited the manuscript. ZXE and TF designed the research project. All authors read and approved the final manuscript.

\section{Funding}

This work was supported by the National Natural Sciences Foundation of China (U1304813), the Science Foundation of the Henan Province of China (20B310016), key R \& D and promotion project in Henan Province (202102310101), and Zhengzhou general science and technology public relations project (141PPTGG449).

\section{Availability of data and material}

All data generated or analyzed during this study are included in this published article.

\section{Ethics approval and consent to participate}


This study was approved by the Review Board and Ethical Committee of Zhengzhou University. All experimental procedures were performed in accordance with the ARRIVE guidelines. All methods were carried out in accordance with relevant guidelines and regulations.

\title{
Consent for publication
}

Not applicable.

\section{Competing interests}

The authors declare that they have no competing interests.

\author{
Author details \\ ${ }^{1}$ The Pathophysiology Department, School of Basic Medical Sciences, Zhengzhou \\ University, Zhengzhou 450001, China; ${ }^{2}$ Provincial Cooperative Innovation Center for \\ Cancer Chemoprevention, Zhengzhou University, Zhengzhou 450001, China; ${ }^{3}$ Cancer \\ Chemoprevention International Collaboration Laboratory, Zhengzhou 450001, China; \\ ${ }^{4}$ Second People's Hospital of Henan Province, Zhengzhou 451191, China; ${ }^{5}$ SanQuan \\ College of XinXiang Medical University, Xinxiang 453003, China; ${ }^{6}$ Center of Genetics \\ and Prenatal Diagnosis, Department of Obstetrics and Gynecology, the First Affiliated \\ Hospital of Zhengzhou University, Zhengzhou 450052, China.
}

\section{References}

[1] Sung H, Ferlay J, Siegel RL, et al. Global Cancer Statistics 2020: GLOBOCAN Estimates of Incidence and Mortality Worldwide for 36 Cancers in 185 Countries. CA Cancer J Clin. 2021;71(3):209-249. https://doi.org/10.3322/caac.21660

[2] Smyth EC, Lagergren J, Fitzgerald RC, et al. Oesophageal cancer. Nat Rev Dis Primers. 2017;3:17048. https://doi.org/10.1038/nrdp.2017.48

[3] Domper A M, Ferrandez A A, Lanas A A. Esophageal cancer: Risk factors, screening and endoscopic treatment in Western and Eastern countries. World J Gastroenterol, 2015,21(26):79337943. https://doi.org/10.3748/wjg.v21.i26.7933

[4] Coleman HG, Xie SH, Lagergren J. The Epidemiology of Esophageal Adenocarcinoma. Gastroenterology. 2018,154(2):390-405. https://doi.org/10.1053/j.gastro.2017.07.046

[5] Arnold M, Soerjomataram I, Ferlay J, et al. Global incidence of oesophageal cancer by histological subtype in 2012. Gut, 2015,64(3):381-387. https://doi.org/10.1136/gutjnl-2014-308124

[6] Yang Z, Zeng H, Xia R, et al. Annual cost of illness of stomach and esophageal cancer patients in urban and rural areas in China: A multi-center study. Chin J Cancer Res, 2018,30(4):439-448. https://doi.org/10.21147/j.issn.1000-9604.2018.04.07

[7] Wang SM, Abnet CC, Qiao YL. What have we learned from Linxian esophageal cancer etiological studies?. Thorac Cancer. 2019,10(5):1036-1042. https://doi.org/10.1111/1759-7714.13058

[8] Wang W L, Chang W L, Yang H B, et al. Low disabled-2 expression promotes tumor progression and determines poor survival and high recurrence of esophageal squamous cell carcinoma. Oncotarget, 2016,7(44):71169-71181. https://doi.org/10.18632/oncotarget.8460 
[9] Wei WQ, Hao CQ, Guan CT, et al. Esophageal Histological Precursor Lesions and Subsequent 8.5Year Cancer Risk in a Population-Based Prospective Study in China. Am J Gastroenterol. 2020,115(7):1036-1044. https://doi.org/10.14309/ajg.0000000000000640

[10] Kosaku M, Leo Y, Daisuke U, et al. Immunotherapy for esophageal squamous cell carcinoma: a review. Fukushima journal of medical science, 2018,64(2):46-53. https://doi.org/10.5387/fms.2018-09

[11] Jin W. Role of JAK/STAT3 Signaling in the Regulation of Metastasis, the Transition of Cancer Stem Cells, and Chemoresistance of Cancer by Epithelial-Mesenchymal Transition. Cells, 2020,9(1):217. https://doi.org/10.3390/cells9010217

[12] Chaluvally-Raghavan P, Jeong K J, Pradeep S, et al. Direct Upregulation of STAT3 by MicroRNA551b-3p Deregulates Growth and Metastasis of Ovarian Cancer. Cell Rep, 2016,15(7):1493-1504. https://doi.org/10.1016/j.celrep.2016.04.034

[13]Furtek S L, Backos D S, Matheson C J, et al. Strategies and Approaches of Targeting STAT3 for Cancer Treatment. ACS Chem Biol, 2016,11(2):308-318. https://doi.org/10.1021/acschembio.5b00945

[14] Fan Y, Mao R, Yang J. NF- $\mathrm{KB}$ and STAT3 signaling pathways collaboratively link inflammation to cancer. Protein Cell. 2013,4(3):176-185. https://doi.org/10.1007/s13238-013-2084-3

[15] Yu H, Lee H, Herrmann A, et al. Revisiting STAT3 signalling in cancer: new and unexpected biological functions. Nat Rev Cancer. 2014,14(11):736-746. https://doi.org/10.1038/nrc3818

[16] Liang C, Zhao T, Li H, et al. Long Non-coding RNA ITIH4-AS1 Accelerates the Proliferation and Metastasis of Colorectal Cancer by Activating JAK/STAT3 Signaling. Mol Ther Nucleic Acids. 2019,18:183-193. https://doi.org/10.1016/j.omtn.2019.08.009

[17] Johnson DE, O'Keefe RA, Grandis JR. Targeting the IL-6/JAK/STAT3 signalling axis in cancer. Nat Rev Clin Oncol. 2018,15(4):234-248. https://doi.org/10.1038/nrclinonc.2018.8

[18] Tian F, Yang X, Liu Y, et al. Constitutive activated STAT3 is an essential regulator and therapeutic target in esophageal squamous cell carcinoma. Oncotarget, 2017,8(51):88719. https://doi.org/10.18632/oncotarget.20838

[19]Liu Y, Wang X, Zeng S, et al. The natural polyphenol curcumin induces apoptosis by suppressing STAT3 signaling in esophageal squamous cell carcinoma. J Exp Clin Cancer Res. 2018,37(1):303. https://doi.org/10.1186/s13046-018-0959-0

[20] Volker L A, Maar B A, Pulido G B, et al. Neph2/Kirrel3 regulates sensory input, motor coordination, and home-cage activity in rodents. Genes Brain Behav, 2018,17(8):e12516. https://doi.org/10.1111/gbb.12516

[21] Roh J D, Choi S Y, Cho Y S, et al. Increased Excitatory Synaptic Transmission of Dentate Granule Neurons in Mice Lacking PSD-95-Interacting Adhesion Molecule Neph2/Kirrel3 during the Early Postnatal Period. Front Mol Neurosci, 2017,10:81. https://doi.org/10.3389/fnmol.2017.00081

[22] Martin E A, Woodruff D, Rawson R L, et al. Examining Hippocampal Mossy Fiber Synapses by 3D Electron Microscopy in Wildtype and Kirrel3 Knockout Mice. eNeuro, 2017,4(3). https://doi.org/10.1523/ENEURO.0088-17.2017

[23] Freddie, Bray, Jacques, et al. Global cancer statistics 2018: GLOBOCAN estimates of incidence and mortality worldwide for 36 cancers in 185 countries. CA: a cancer journal for clinicians, 2018,68(6):394-424. https://doi.org/10.3322/caac.21492

[24] Yang S, Lin S, Li N, et al. Burden, trends, and risk factors of esophageal cancer in China from 1990 to 2017: an up-to-date overview and comparison with those in Japan and South Korea. J Hematol Oncol. 2020,13(1):146. https://doi.org/10.1186/s13045-020-00981-4

[25]Zeng H, Zheng R, Zhang S, et al. Esophageal cancer statistics in China, 2011: Estimates based on 177 cancer registries. Thorac Cancer, 2016,7(2):232-237. https://doi.org/10.1111/1759-7714.12322

[26] GBD 2017 Oesophageal Cancer Collaborators. The global, regional, and national burden of oesophageal cancer and its attributable risk factors in 195 countries and territories, 1990-2017: a systematic analysis for the Global Burden of Disease Study 2017. Lancet Gastroenterol Hepatol. 2020,5(6):582-597. https://doi.org/10.1016/S2468-1253(20)30007-8

[27] Luo L N, He L J, Gao X Y, et al. Evaluation of preoperative staging for esophageal squamous cell carcinoma. World J Gastroenterol, 2016,22(29):6683-6689. https://doi.org/10.3748/wjg.v22.i29.6683

[28]Zeng H, Chen W, Zheng R, et al. Changing cancer survival in China during 2003-15: a pooled analysis of 17 population-based cancer registries. Lancet Glob Health, 2018,6(5): e555-e567. https://doi.org/10.1016/S2214-109X(18)30127-X

[29]Zhang H X, Yang P L, Li E M, et al. STAT3beta, a distinct isoform from STAT3. Int J Biochem 
Cell Biol, 2019,110:130-139. https://doi.org/10.1016/j.biocel.2019.02.006

[30] Li P, Huang T, Zou Q, et al. FGFR2 Promotes Expression of PD-L1 in Colorectal Cancer via the JAK/STAT3 Signaling Pathway. J Immunol, 2019,202(10):3065-3075. https://doi.org/10.4049/jimmunol.1801199

[31]Liang Q, Ma D, Zhu X, et al. RING-Finger Protein 6 Amplification Activates JAK/STAT3 Pathway by Modifying SHP-1 Ubiquitylation and Associates with Poor Outcome in Colorectal Cancer. Clin Cancer Res, 2018,24(6):1473-1485. https://doi.org/10.1158/1078-0432.CCR-17-2133

[32]Feng T, Cao W, Shen W, et al. Arctigenin inhibits STAT3 and exhibits anticancer potential in human triple-negative breast cancer therapy. Oncotarget, 2017,8(1):329-344. https://doi.org/10.18632/oncotarget.13393

[33] Shi S, Zheng G, Yang C, et al. Effects of Vitamin K3 Combined with UVB on the Proliferation and Apoptosis of Cutaneous Squamous Cell Carcinoma A431 Cells. Onco Targets Ther, 2019,12:11715-11727. https://doi.org/10.2147/OTT.S228792

[34] Wang Z, Liu W, Wang C, et al. miR-873-5p Inhibits Cell Migration and Invasion of Papillary Thyroid Cancer via Regulation of CXCL16. Onco Targets Ther, 2020,13:1037-1046. https://doi.org/10.2147/OTT.S213168

[35] Heikenwalder M, Lorentzen A. The role of polarisation of circulating tumour cells in cancer metastasis. Cell Mol Life Sci, 2019,76(19):3765-3781. https://doi.org/10.1007/s00018-019-031693

[36]Zhang Y, Zhang M, Fan X, et al. Effect of STAT3 decoy oligodeoxynucleotides mediated by ultrasound-targeted microbubbles combined with ultrasound on the growth of squamous cell carcinoma of the esophagus. Oncol Lett, 2019,17(2):2151-2158. https://doi.org/10.3892/ol.2018.9814 